\title{
Determination of the Efficiencies of the Prokinetics in Ruminants with Postoperative Ileus Using Pro-Inflammatory Markers
}

\author{
Semih Altan', Kaan Dönmez², Feray Altan³ \& Fahrettin Alkan²
}

\begin{abstract}
Background: Recently, the role of inflammation triggered by handling of the intestine various gastrointestinal (GI) surgeries is generally accepted as the key event in postoperative ileus (POI). Because, prokinetics have been increased the smooth muscle contractions and may act by attenuating the inflammatory process in the GI tract, they have been used the treatment of POI in human and animals. There are many in vivo analysis techniques of GI motility. However, there have not yet been studied associated with the evaluation of the inflammatory response. Therefore, it was aimed to evaluate the efficiencies of 3 different prokinetics from inflammatory response during experimentally-induced POI.

Materials, Methods \& Results: Twenty healthy lambs (30-45 days old) were randomly assigned to four groups. In all groups, enterotomy was performed on the ileum. Erythromycin and metoclopramide were administered to the ERT and MET groups before the surgery, respectively, while lidocaine was administered to the LID group as bolus before and continuous rate infusion during the surgery. Physiological saline was administered to the lambs in control group as placebo before the surgery. Blood samples were collected before surgery $(\sim 30-45 \mathrm{~min})$, at the end of surgery $(0 \mathrm{~h})$, and at the postoperative 1, 3, 5, 10, 48, 72 and $96 \mathrm{~h}$. The concentrations of serum amyloid A (SAA), haptoglobin (HPT), fibrinogen (FIB) as acute phase proteins (APPs), thiobarbituric acide reactant substrate (TBARs), myeloperoxidase (MPO) as reactive oxygen species, and transforming growth factor-beta (TGF- $\beta$ ) as a cytokine were measured with ELISA reader. In terms of time points, it was found that FIB was statistically higher in ERT group at the 1st $\mathrm{h}$, in MET and LID groups at the 10th $\mathrm{h}$, and in LID group at the 48th and in MET group at the $72 \mathrm{~h}(P<0.05)$. It was found that SAA was higher in MET group at the 1st, 3rd, 5th, 10th, 24th, 48th and 72nd h. HPT was higher in CNTR group until 72th $\mathrm{h}$ and MET group at 48th, 72nd and 96th $\mathrm{h}$. TBARs concentrations were statistically higher in MET and LID groups at 0 hour, in ERT and MET groups at the $1 \mathrm{st} \mathrm{h}$, in MET group at the 3rd h, in MET and LID groups at the 5th and 10th h, in MET group at the 48th, 72nd and 96th $\mathrm{h}$ $(P<0.05)$. MPO concentrations was higher in LID group at the 3rd, 5th, 10th and 96th $\mathrm{h}$, and in ERT group at the $72 \mathrm{nd} \mathrm{h}$ $(P<0.05)$. TGF- $\beta$ concentrations were particularly high in MET group at the 3rd, 5th, 48th and $72 \mathrm{nd} \mathrm{h}$, and in LID group at the 10th, 24th, and 96th $\mathrm{h}(P<0.05)$.

Discussion: APPs (HPT, SAA, FIB), which are important regulators of inflammation in cows and sheep, were higher generally in MET and LID groups and inflammation persists in these two groups and, therefore, metoclopramide and lidocaine are less effective in early postoperative POI treatment. Because, significant increase in serum TBARs and MPO concentrations was considered as an important indicator of oxidative stress and inflammatory response MPO concentrations was particularly high until 10th $\mathrm{h}$ in LID group, and TBARs concentrations was high both MET and LID groups throughout the study, this was correlated with higher neutrophil infiltration in the postoperative early period than the other groups. It is known that TGF- $\beta$, an inflammatory cytokine, is correlated with various smooth muscle disorders in humans. In this study, TGF- $\beta$ concentration were higher in the MET and LID groups. High concentration of this cytokine might have led to decrease contractions in smooth muscles, thereby slowing down the intestinal transition. In conclusion, based on the presence of pro-inflammatory markers in this study, erythromycin seems to be the most suitable prokinetic drug in lambs. Moreover, lidocaine and metoclopramide are not as successful in small ruminants as reported in other species.
\end{abstract}

Keywords: postoperative ileus, pro-inflammatory markers, prokinetic drugs, ruminant. 


\section{INTRODUCTION}

Recently, the role of inflammation triggered by handling of the intestine various gastrointestinal (GI) surgeries is generally accepted as the key event in postoperative ileus (POI) [12,27]. It is thought that various mechanisms such as sympathetic neural reflexes, local or systemic inflammatory mediators [cytokines, reactive oxygen species (ROS), acute phase proteins (APP)] and changes in the neural and hormonal transmitter levels play a role occurrence of POI and that recovery occurs by the inhibition of these mediators. It has become clear that inflammatory mediators released by leucocytes within the gut wall also directly impair smooth muscle contractility $[3,17,26]$.

Prokinetic drugs used treatment of the POI, which increased the smooth muscle contractions in the GI tract, may act by attenuating the inflammatory process $[8,18,20]$. Owing to these features, they have been used the treatment of POI in human and animals. Although, many of these drugs have been used successfully in monogastric animals, they are limited numbers in the ruminant. Bethanechol, neostigmine, metoclopramide, erythromycin and lidocaine are used in ruminants. However, except erythromycin, the effectiveness of these drugs is not clearly addressed in ruminant $[1,18,23]$.

There are many in vivo analysis techniques of GI motility $[6,14,18]$. However, there have not yet been studied associated with the evaluation of the inflammatory response concerning in ruminants with POI. Therefore, it was aimed to evaluate the efficiencies of 3 different prokinetics -erythromycin, lidocaine and metoclopramide- from systemic inflammatory response during experimentally-induced POI by studying APPs, ROS and cytokines.

\section{MATERIALS AND METHODS}

\section{Animals}

In the study, 20 male lambs of Kivircik breed, aged 30-45 days, body weight of 8.7-15.6 kg (mean $12.35 \mathrm{~kg}$ ) were used. After routine clinical examination (appetite, body temperature, pulse, respiratory rate, defecation), healthy lambs were included in the study. Lambs were fed with standard lamb feed (ad libitum) and they always had access to water end of the study.
Study groups

The study was performed with 4 groups, each randomly assigned 5 lambs; CNTR was set as the control group. Surgery was performed on the lambs in this group and $2 \mathrm{~mL}$ physiological saline $(0.9 \%$ $\mathrm{NaCl}$ ) as placebo was administered IM before the surgery. In the ERT group, lambs were administered with $8.8 \mathrm{mg} \mathrm{kg}^{-1}$ erythromycin (Apirocin- $\left.{ }^{\circledR}\right)^{1}$. intramuscularly before the surgery. In the MET group, lambs were injected with $0.2 \mathrm{mg} \mathrm{kg}^{-1}$ metoclopramide $\left(\text { Metpamid }^{\circledR}\right)^{2}$ intramuscularly before the surgery. In the LID group, before the surgery, $1.3 \mathrm{mg}$ $\mathrm{kg}^{-1}$ lidocaine hydrochloride $\left(\text { Vilcain }^{\circledR}\right)^{3}$ bolus was given intravenously, and then, during the surgery, $0.05 \mathrm{mg} / \mathrm{kg} / \mathrm{h}$ was given intravenously as continuous rate infusion (CRI).

\section{Preparation of lambs for the surgery and anesthesia protocol}

Food was withheld $12 \mathrm{~h}$ prior to surgery in each lamb, and only water was provided. The area between the umbilicus and pelvic region was shaved for surgery. General anesthesia was performed by injecting $6 \mathrm{mg} \mathrm{kg}^{-1}$ ketamine hydrochloride (Keta$\left.\mathrm{sol}^{\circledR}\right)^{4}$ intramuscularly following the injection of $0.2 \mathrm{mg} \mathrm{kg}^{-1}$ xylazine hydrochloride $\left(\operatorname{Rompun}^{\circledR}\right)^{5}$ as intramuscularly.

\section{Surgical procedure for POI model}

Lambs were restrained in dorsal recumbency. Surgical area was aseptically prepared. Laparotomy was performed through a $10 \mathrm{~cm}$-long paramedian incision made caudally to the umbilicus along the preputium. Once the organs in the abdominal cavity were checked, ileum was reached. Ileum was held between the thumb and index finger and pulled out from the incision line. Ileal contents were moved towards the caudal region by gentle squeezing, and intestinal forceps were placed distally and proximally to the enterotomy area and a $5 \mathrm{~cm}$-long longitudinal incision was made at the antimesenteric side of the ileum. Enterotomy area, which was sanitized with a wet gauze, was closed with continuous double needle sutures using 3/0 polydioxanone. After the sutured area was sanitized with a wet gauze and checked for any leakages, forceps were removed, intestinal segment was released to the abdominal cavity and abdominal wall was closed routinely. 


\section{Blood sampling and laboratory measurements}

An intravenous catheter was placed in $V$. jugularis of the lambs, and blood was collected before and following surgery at $0,1,3,5,10,48,72$ and 96th h. Collected blood samples were centrifuged at $2500 \mathrm{~g}$ for $10 \mathrm{~min}$ and serum samples were obtained. Concentrations of the serum haptoglobin (HPT), Fibrinogen (FIB), serum amyloid A (SAA), thiobarbutiric acid reactant substances (TBARs), myeloperoxidase (MPO), and transforming growth factor-beta (TGF- $\beta$ ) were determined by ELISA/spectrophotometer reader (Multiskan Go Spectrophotometer) ${ }^{6}$ according to the sheep-specific ELISA kits ${ }^{7}$ protocol.

\section{Statistical analysis}

Statistical analysis was performed by the Statistical Package for Social Sciences (SPSS) v20.0 software ${ }^{8}$. The obtained data were evaluated by variance analysis performed using one-way analysis of variance ANOVA and Tukey post hoc test. Data were expressed as mean $\pm \mathrm{SD}$ and $P<0.05$ was considered as statistically significant difference.

\section{RESULTS}

The mean concentrations of acute phase proteins (HPT, SAA and FIB), oxidative stress parameters (TBARs, MPO) and cytokine (TGF- $\beta$ ) among the study groups are presented in Table 1.

\section{Acute phase proteins}

In terms of APP, it was found that HPT, SAA and FIB values increased at the end of the surgery in all groups except MET group. In terms of time points, it was found that FIB was statistically higher in ERT group at the 1st h, in MET and LID groups at the 10th $\mathrm{h}$, and in LID group at the 48th and in MET group at the 72 nd h $(P<0.05)$. It was found that SAA was higher in MET group at the 1st, 3rd, 5th, 10th, 24th, 48th and 72nd h. HPT was higher in CNTR group until 72th h and MET group at 48th, 72nd and 96th $\mathrm{h}$.

\section{Oxidative stress parameters}

TBARs concentrations were statistically higher in MET and LID groups at $0 \mathrm{~h}$, in ERT and MET groups at the 1st h, in MET group at the 3rd h, in MET and LID groups at the 5th and 10th $\mathrm{h}$, in MET group at the 48th, 72nd and 96th $\mathrm{h}(P<0.05)$. MPO concentrations was higher in LID group at the 3rd, 5th, 10th and 96th $\mathrm{h}$, and in ERT group at the 72nd $\mathrm{h}(P<0.05)$.

\section{Cytokine concentration}

TGF- $\beta$ concentrations were particularly high in MET group at the 3rd, 5th, 48th and 72nd h, and in LID group at the 10th, 24th, and 96th $\mathrm{h}(P<0.05)$.

\section{DISCUSSION}

HPT, with SAA in particular, is a major APP in lambs [4], and has antioxidant, anti-inflammatory, immune regulatory and antibacterial properties. Therefore, it can be used to detect various diseases $[4,13]$. In this study, HPT concentrations obtained in the measurement of immediately after the surgery $(0$ h) was higher in MET and LID groups, and these high levels sustained throughout the first $10 \mathrm{~h}$ particularly in the MET group. In addition, from the post-operative $1 \mathrm{st} \mathrm{h}$ until the 72th $\mathrm{h}$, SAA concentrations were higher in the MET group. The observed increases in HPT and SAA concentrations in the MET group support the fact that inflammation persists and, therefore, metoclopramide is less effective in POI treatment. On the other hand, the increases in the SAA and HPT concentrations in the LID group similarly to the MET group can be considered as evidences that the inflammation persists after the administration of lidocaine. As the concentrations of fibrinogen are important regulators of inflammation in cows and sheep [4,9], it is reported as a reliable indicator of inflammation and bacterial infection [29]. It is known that in acute cases, FIB concentration usually increases within the first $24 \mathrm{~h}$ after tissue damage, reaches its peak levels in 3-4 days, and gradually decreases over time [9]. In all groups, FIB concentration increased during the surgery and its concentration were sustained until the 48th $\mathrm{h}$. Particularly in the MET group, this concentration was statistically higher after the 48th h, suggesting that the acute phase response sustained in this group.

MPO is a marker of neutrophil infiltration that involved in the early stages of inflammation in tissues and is considered to play an important role in the antimicrobial defense mechanism. On the other hand, MPO contributes to oxidative tissue damage during chronic inflammation $[2,24]$. It was reported that the increase in MPO concentrations in cases when motility is disrupted, such as the displacement of abomasum to the left in cows, is correlated with the intensity of necrosis, neutrophil response, and inflammation in the tissue [9]. In this study, MPO concentrations was particularly high in 3rd, 5th and 10th h in LID group, 


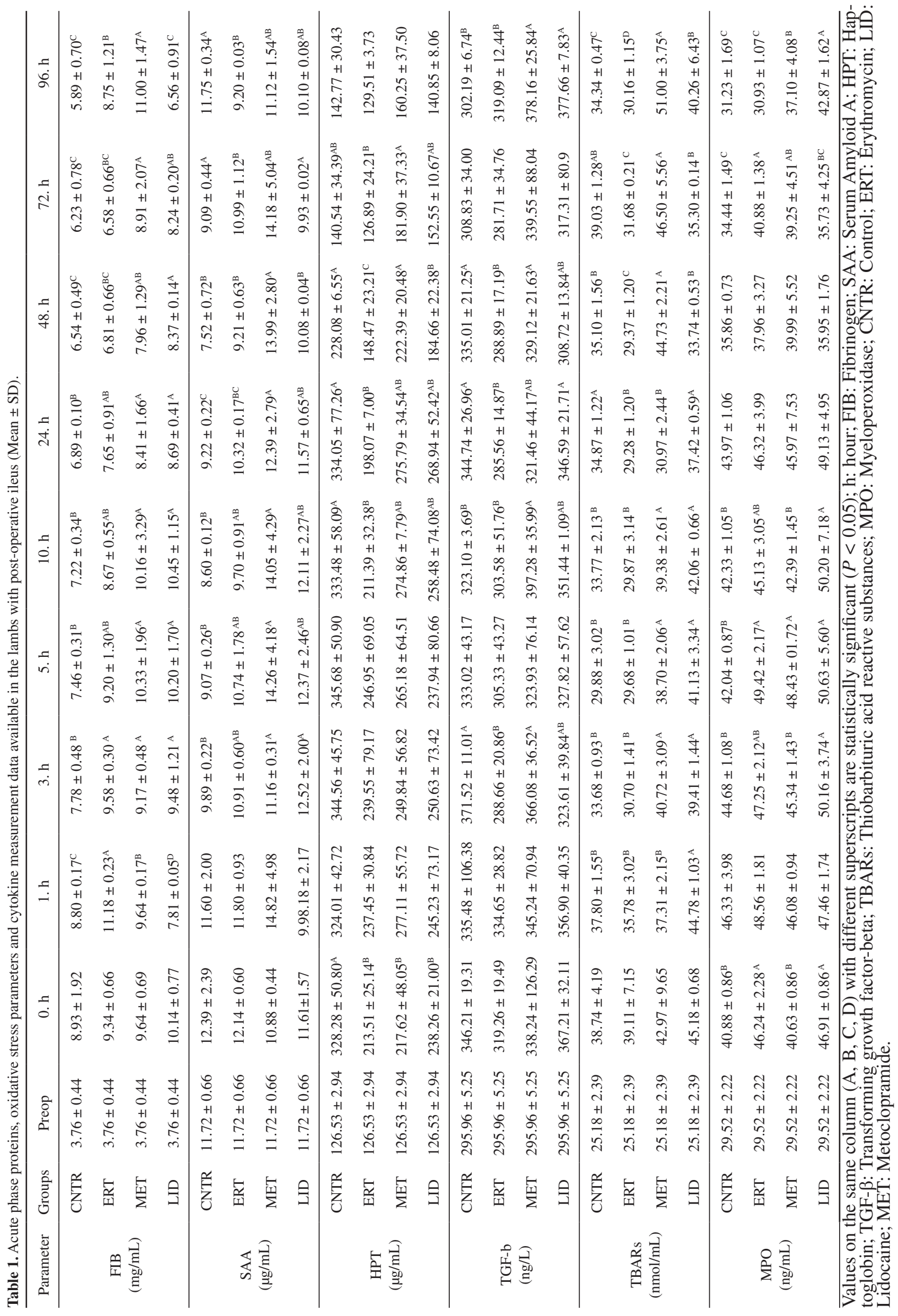


this was correlated with higher neutrophil infiltration in the postoperative early period than the other groups, and this is consistent with the Amanzada et al. [2]. TBARs are used to determine the level of oxidative stress in body fluids [5]. In experimentally established abdominal compartment syndrome in rats, significant increase in serum TBARs and MPO concentrations was considered as an important indicator of oxidative stress and inflammatory response [25]. In this study, higher concentrations of TBARs within the first $24 \mathrm{~h}$ in MET and LID groups suggest that inflammation in these groups lasted shorter than the other groups and therefore, POI persisted. In addition, continuing high concentrations of TBARs in the MET group in 48th, 72nd and 96th $\mathrm{h}$ suggested that inflammatory process still persisted in this group.

Cytokines play role in immune system regulation, inflammatory response, cell division/differentiation, wound healing and hematopoiesis [7]. They can also regulate smooth muscle function either by acting directly on smooth muscle cells or indirectly via mediators released from other immune or non-hematopoietic cells. It is known that TGF- $\beta$, an inflammatory cytokine, is correlated with various smooth muscle disorders in humans $[16,22]$. In this study, TGF- $\beta$ concentration were higher in the MET and LID groups. High concentration of this cytokine might have led to decrease contractions in smooth muscles [16], thereby slowing down the intestinal transition.

Erythromycin, a motilin agonist, binds to neurons of the enteric nervous system and to specific receptors found on the intestinal smooth muscles, and acts to increase the contractile activity. It was found that high doses of ( $\left.8.8 \mathrm{mg} \mathrm{kg}^{-1} \mathrm{IM}\right)$ erythromycin in healthy calves increases frequency through abomasal intraluminal pressure and speeds up the abomasal emptying time [19,30]. In this study, decrease in APPs, and recovery of oxidative damage markers (TBARs and MPO) faster than the other groups may suggest that erythromycin is significantly effective in POI in lambs as it is in other animal species.

Although some researchers reported that the use of metoclopramide, which shows its prokinetic effect by increasing acetylcholine release from enteric cholinergic neurons, is not effective in POI in animals and humans [21,30], some researchers proposed that it is effective, since it causes a temporary increase in the contractile and myoelectrical activity of the proximal
GI system in dogs [10] and in the electrical activity of pyloric antrum in goats [11]. In this study, as the metoclopramide group was worse in APPs, oxidative damage and cytokine terms than the other groups, it was considered to be not as effective as desired in POI in lambs similar to Seta \& Kalle-Pradhan [21] and Wittek \& Constable [30].

The effects of lidocaine on GI system are thought to be exerted via the reduction of circulating catecholamines, prevention of the formation of free radicals, anti-inflammatory properties, blocking the sympathetic effects, and stimulation of parasympathetic reflexes [28]. Van Hoogmoed et al. [28] reported that veterinary surgeons have $1.3 \mathrm{mg} \mathrm{kg}^{-1}$ lidocaine bolus alone as the primary option, or subsequently use at a rate of $0.5 \mathrm{mg} \mathrm{kg}^{-1} / \mathrm{min}$ as CRI. On the other hand, Milligan et al. [15] suggested that using $1.3 \mathrm{mg} \mathrm{kg}^{-1}$ lidocaine bolus followed by using at a rate of $0.5 \mathrm{mg}$ $\mathrm{kg}^{-1} / \mathrm{min}$ for $3 \mathrm{~h}$ is not effective as a prokinetic drug in healthy horses, but can give different results in horses with various GI problems. In this study, based on the concentrations of APPs, oxidative damage markers and cytokine, it was suggested that the use of lidocaine (1.3 $\mathrm{mg} \mathrm{kg}^{-1}$ bolus followed by $0.05 \mathrm{mg} \mathrm{kg}^{-1} / \mathrm{min}$ as CRI) for POI treatment in lambs cannot be an alternative for erythromycin in terms of its prokinetic properties.

\section{CONCLUSIONS}

In conclusion, based on the presence of proinflammatory markers in this study, erythromycin seems to be the most suitable prokinetic drug in lambs. Moreover, lidocaine and metoclopramide are not as successful in small ruminants as reported in other species.

\section{MANUFACTURERS}

${ }^{1}$ Teknovet İlaç Sanayi. Istanbul, Turkey.

${ }^{2}$ Recordati İlaç Sanayi. Istanbul, Turkey.

${ }^{3}$ Vilsan İlaç Sanayi. Ankara, Turkey.

${ }^{4}$ Richterpharma AG. Wells, Austria.

${ }^{5}$ Bayer Animal Health. Istanbul, Turkey.

${ }^{6}$ Thermo Fisher Scientific Inc. Hudson, NH, USA.

${ }^{7}$ Sunred Biological Technology Co. Ltd. Shangai, China. ${ }^{8}$ IBM Corp. Armonk, NY, USA.

Acknowledgements. This research was financially supported by the Scientific Research Council of Dicle University (Project No: Veteriner.15.002) and a part study was orally presented in the 1st ICAVST congress in Sarajevo/Bosnia-Herzegovina between 25 and 29 August 2016. 
Ethical approval. All procedures, treatments and animal care were approved by Animal Experiments Local Ethical Committee of Dicle University (No:2014/059).
Declaration of interest. The authors report no conflicts of interest. The authors alone are responsible for the content and writing of paper.

\section{REFERENCES}

1 Abell T.L., Bernstein R.K., Cutts T., Farrugia G., Forster J., Hasler W.L., McCallum R.W., Olden K.W., Parkman H.P., Parrish C.R., Pasricha P.J., Prather C.M., Soffer E.E., Twillman R. \& Vinik A.I. 2006. Treatment of gastroparesis: a multidisciplinary clinical review. Neurogastroenterology and Motility. 18: 263-283.

2 Amanzada A., Malik I.A., Nischwitz M., Sultan S., Naz N. \& Ramadori G. 2011. Myeloperoxidase and elastase are only expressed by neutrophils in normal and in inflamed liver. Histochemistry and Cell Biology. 135: 305-315.

3 Boeckxstaens G.E. \& de Jonge W.J. 2009. Neuroimmune mechanisms in postoperative ileus. Gut. 58: 1300-1311.

4 Chemonges S., Tung J.P. \& Fraser J.F. 2014. Proteogenomics of selective susceptibility to endotoxin using circulating acute phase biomarkers and bioassay development in sheep: a review. Proteome Science. 1: 12.

5 Chi C.H., Shiesh S.C. \& Lin X.Z. 2002. Total antioxidant capacity and malondialdehyde in acute abdominal pain. The American Journal of Emergency Medicine. 20: 79-82.

6 Constable P., Hinchcliff K., Stanley H. \& Grünberg W. 2017. Diseases of the Forestomach of Ruminants. In: Constable P., Hinchcliff K., Stanley H. \& Grünberg W. (Eds). Veterinary Medicine: A textbook of the diseases of cattle, horses, sheep, pigs and goats. 11th edn. Philadelphia: Elsevier, pp.435-621.

7 Corum O., Er A. \& Dik B. 2016. Investigation of the effect of tarantula cubensis extract on acute phase response. Acta Scientiae Veterinariae. 44: 1414.

8 Dowling P.M. 1995. Prokinetic drugs: metoclopramide and cisapride. Canadian Veterinary Journal. 36(2): 115-116.

9 Gökçe H.İ. \& Bozukluhan K. 2009. Çiftlik hayvanlarında önemli akut faz proteinleri ve bunların veteriner hekimlik alanındaki kullanımı. Dicle Üniversitesi Veteriner Fakültesi Dergisi. 1: 1-14.

10 Graves G.M., Becht J.L. \& Rawlings C.A. 1989. Metoclopramide reversal of decreased gastrointestinal myoelectric and contractile activity in a model of canine postoperative ileus. Veterinary Surgery. 18: 27-33.

11 Huhn J.C. \& Nelson D.R. 1997. The quantitative effect of metoclopramide on abomasal and duodenal myoelectric activity of goats. Zentralblatt für Veterinärmedizin. Reihe A 44: 361-371.

12 Johnson M.D. \& Walsh R.M. 2009. Current therapies to shorten postoperative ileus. Cleveland Clinic Journal of Medicine. 76: 641-648.

13 Maden M., Ozturk A.S., Bulbul A., Avci G.E. \& Yazar E. 2012. Acute-phase proteins, oxidative stress and enzyme activities of blood serum and peritoneal fluid in cattle with abomasal displacement. Journal of Veterinary Internal Medicine. 26: 1470-1475.

14 Marshall T.S., Constable P.D., Cronchik S.S. \& Wittek T. 2005. Determination of abomasal emptying rate in suckling calves by use of nuclear scintigraphy and asetominophen absorption. American Journal of Veterinary Research. 66: 364-374.

15 Milligan M., Beard W., Kukanich B., Sobering T. \& Waxman S. 2007. The effect of lidocaine on postoperative jejunal motility in normal horses. Veterinary Surgery. 36: 214-220.

16 Moore-Olufemi S.D., Olsen A.B., Hook-Dufresne D.M., Bandla V. \& Cox Jr. C.S. 2015. Transforming growth factor-beta 3 alters intestinal smooth muscle function: implications for gastroschisis-related intestinal dysfunction. Digestive Diseases and Sciences. 60: 1206-1214.

17 Navarre C.B. \& Roussel A.J. 1996. Gastrointestinal motility and disease in large animals. Journal of Veterinary Internal Medicine. 10: 51-59.

18 Ness S.L. \& Steiner A. 2017. Motility modifiers. In: Fubini S.L. \& Ducharme N.G. (Eds). Farm Animal Surgery. 2nd edn. St. Louis: Elsevier, pp.89-98.

19 Nouri M. \& Constable P.D. 2007. Effect of parenteral administration of erythromycin, tilmicosin and tylosin on abomasal emptying rate in suckling calves. American Journal of Veterinary Research. 68(12): 1392-1398.

20 Reynolds J.C. 1989. Prokinetic agents: a key in the future gastroenterology. Gastroenterology Clinics of North America. 18: 437-457.

21 Seta M.L. \& Kale-Pradhan P.B. 2001. Efficacy of metoclopramide in postoperative ileus after exploratory laparotomy. Pharmacotherapy. 21: 1181-1186. 
22 Shea-Donohue T., Notari L., Sun R. \& Zhao A. 2012. Mechanisms of smooth muscle responses to inflammation. Neurogastroenterology and Motility. 24: 802-811.

23 Smith M.A., Edwards G.B., Dallap B.L., Cripps P.J. \& Proudman C.J. 2005. Evaluation of the clinical efficacy of prokinetic drugs in the management of postoperative ileus: can retrospective data help us. The Veterinary Journal. 170: 230-236.

24 Sokolov A.V., Pulina M.O., Ageeva K.V., Ayrapetov M.I., Berlov M.N., Volgin G.N., Markov A.G., Yablonsky P.K., Kolodkin N.I., Zakharova E.T. \& Vasilyev V.B. 2007. Interaction of ceruloplasmin, lactoferrin, and myeloperoxidase. Biochemistry. 72: 409-415.

25 Tihan D.C., Erbil Y., Seven R., Arkaya S., Türkoğlu U., Hepgül G. \& Borucu I. 2011. The effect of glutamine on oxidative damage in an experimental abdominal compartment syndrome model in rats. Ulusal Travma ve Acil Cerrahi Dergisi. 17: 1-8.

26 Tropskaya N.S. \& Popova T.S. 2017. Postoperative ileus: Pathophysiology and Treatment. In: Lule G. (Ed). Current concepts in colonic disorders. Intech Publisher open access pp.203-220. http://library.umac.mo/ebooks/b28358740. pdf. [Accessed online in September 2017].

27 Van Bree S.H., Nemethova A., Van Bovenkamp F.S., Gomez-Pinilla P., Elbers L., Di Giovangiulio M., Matteoli G., Van Vliet J., Cailotto C., Tanck M.W. \& Boeckxstaens G.E. 2012. Novel method for studying postoperative ileus in mice. International Journal of Physiology, Pathophysiology and Pharmacology. 4: 219-227.

28 Van Hoogmoed L.M., Nieto J.E., Snyder J.R. \& Harmon F.A. 2004. Survey of prokinetic use in horses with gastrointestinal injury. Veterinary Surgery. 33: 279-285.

29 Wang S., Liu J., Wu D.I., Pang X., Zhao J. \& Zhang X. 2015. Pro-inflammatory effect of fibrinogen on vascular smooth muscle cells by regulating the expression of PPAR $\alpha$, PPAR $\gamma$ and MMP-9. Biomedical Reports. 3: 513-518.

30 Wittek T. \& Constable P.D. 2005. Effect of erythromycin, neostigmine and metoclopramide on abomasal motility and emptying rate in Holstein-Freisian calves. American Journal of Veterinary Research. 66: 545-552. 